\section{Mechanism of T-cell tolerance}

\author{
from Roger B. Taylor
}

IN spite of the burgeoning literature on suppressor cells and immunological networks, there has been persistent support for the notion that responsive lymphoid cells may be specifically deleted. This term is now broadly interpreted to mean some internal change occurring in the cell to render it either temporarily or permanently inactive.

We already know that B cells, especially in their immature stages, can enter a condition of lasting unresponsiveness sometimes accompanied by a defect in the expression of receptors. So far, however, the evidence for deletion of helper $T$ cells has been restricted to experiments in vivo, almost entirely in mice, using mammalian serum proteins in high dosage as tolerogens.

To be certain that no suppressor influences are at work one should ideally study tolerance in monotypic cell populations, and preferably clones, in vitro. It was therefore a breakthrough, important in more than one sense, when a group working at University College, London, recently achieved the induction of tolerance in human helper T-cell clones in vitro' .

These clones were specific for peptides derived from the influenza haemagglutinin molecule, and were propagated by stimulation with the specific peptide in the presence of unseparated human mononuclear cells which served as antigenpresenting cells. After such stimulation the cells would both exhibit a proliferative response and help B cells to produce antibody. Lamb and his co-workers then tried incubating the clomes with high concentrations of the peptides, in the absence of the human mononuclear cells. On subsequently testing the response to the usual low stimulatory concentrations in the presence of the human mononuclear cells they found that the $T$ cells had become specifically unresponsive. The tolerized cells would continue to divide in the presence of interleukin- 2 but only for a period of 1-2 weeks, after which their viability declined. A critical finding was that the 'tolerized' cells had lost the surface marker T3 which is thought to be closely associated with the antigen receptor ${ }^{2}$. It confirmed that some demonstrable change had occurred in the cells, and thus argued that the tolerance was not due merely to blocking of the receptors by the high concentration of peptide. A parallel finding was made by Schlossman's group in Boston, who induced a similar state of deletion in human $T$ cells by means not of antigen but of anti-receptor antibodies ${ }^{3}$.

Against this background, interest now focuses on the mechanism by which such deletion comes about. A pertinent report has recently appeared from Schwartz's group at the National Institutes of Health, on cloned mouse $T$ cells ${ }^{4}$. These clones, specific for pigeon cytochrome $c$, were proirradiated mouse spleen cells or a B-lymphoma cell line as antigen-presenting cells. The proliferative response was measured in the presence of varying concentrations of both antigen and the relevant Ia. (The latter was varied by changing the numbers of presenting cells, blocking with anti-Ia antibody, or by the choice of cells homozygous or heterozygous for the at high concentrations of either antigen or Ia or of both. A clear relationship emerged whereby the response depended on the product of the concentrations of antigen and of Ia. One implication is that a physical product of these two molecules must be the critical driving force for T-cell activation. For the high-dose inhibition to be classed as tolerance, it would need to render the cells refractory to subsequent stimulation with optimal doses. This has not yet been reported. Nevertheless the similarity to the human T-cell work is obvious.

The question now arising is how the $T$ cell is inhibited. Does the occupation of a high proportion of receptors release a negative signal to the cell interior? Or does intense stimulation cause the cell to release critical amounts of an external feedback signal? The authors suggest that immune interferon could serve as such a feedback pagated by stimulation with this antigen on relevant Ia.) Bell-shaped curves were obtained, in which the response was inhibited

control - particularly as its production is not subject to the same degree of inhibition at high doses of antigen as the proliferative response 5 . There is no evidence so far, however, that the inhibitory effects of interferon on immune responses can persist long enough to be classed as tolerance.

Interferon can also enhance immune responses, as exemplified by a paper in this issue of Nature ${ }^{6}$ (see p.381). It is shown that interferon both increases antibody responses in vivo, and also increases the expression of Ia on an antigen-presenting macrophage cell line. The authors suggest that the effect of interferon on the antibody response is achieved by virtue of its effect on Ia expression. If this is the case, then the bell-shaped curve derived by Schwartz and colleagues leads us to expect that, at high concentrations of antigen, interferon should aid the induction of tolerance rather than immunity. Its activity might then be seen as to shift the curve to the left, and so influence the quantity of signal delivered via the receptors rather than acting itself as a negative signal. This might also be the role of a variety of other 'adjuvant' influences, many of which can have either positive or negative effects in different circumstances.

Roger B. Taylor is in the Department of Pathology, University of Bristol, The Medical School, University Walk, Bristol BS8 ITD.

\footnotetext{
1. L.amb, J.R., Skidmore, B.J., Chiller, J.M. \& Feldmann, M. J. exp. Med. 157, 143 (1983)

2. Lamb, J.R. \& Feldmann, M. Nature 300,456 (1982).

3. Meuer, S.C. et al. J. exp. Med. 157, 705 (1983).

4. Matis, L.A., Glimcher, L..H., Paul, W.E. \& Schwartz, R.H. Proc. natn. Acad. Sci. U.S.A. 80, 6019 (1983).

5. Hecht, T.T., Longo, D.L. \& Matis, L.A. J. Immun. (in the press).

6. Nakamura, M., Manser, T., Pearson, G.D.N., Dakey, M.J. \& Gefter, M.L.. Nature 306, 381 (1984).
}

\section{Latest view of Mount St Helens}
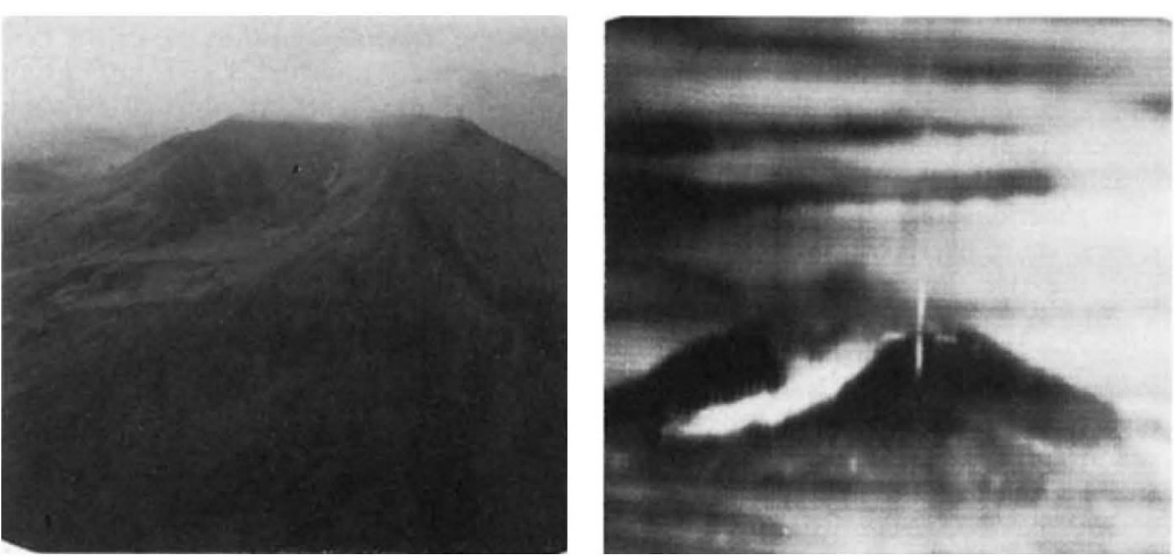

MounT St Helens photographed during its eruption on 18 May 1980 in normal light (top), and in a recently-released US Navy infrared image. White areas on the infrared image represent hotter areas of the volcano and allow the lava flow near the crater and thermal events not usually seen by the naked eye to be detected. The infrared image was taken from a Hughes A-6E Intruder aircraft using its 'Detecting and Ranging Set', a thermal imaging sensor which, when not photographing volcanoes, helps the Intruder's aircrew see and attack surface targets at night or through smoke and haze. $\square$ 